\section{Karlijn THOONEN ${ }^{1}$ \\ Valeria LIMA PASSOS ${ }^{2}$ \\ Francine SCHNEIDER ${ }^{1}$ \\ Hein DE VRIES ${ }^{1}$ \\ Liesbeth VAN OSCH ${ }^{1}$}

${ }^{1}$ Department of Health Promotion, School CAPHRI, Maastricht University,

Maastricht, the Netherlands

${ }^{2}$ Department of Methodology and Statistics,

School CAPHRI, Maastricht University,

Maastricht, the Netherlands

Reprints: Karlijn Thoonen

$<$ karlijn.thoonen@maastrichtuniversity.nl>

Article accepted on 02/02/2021

\title{
Children's sunburn exposed: identification of sun exposure and parental sun protection patterns
}

\begin{abstract}
Background: Preventing sunburn in childhood is imperative in the light of skin cancer prevention. To provide directions for targeted interventions, a better understanding of children's sunburn and associated parental behaviours is necessary. Objectives: To explore sun exposure and parentfor-child sun protection patterns and their relationship with sunburn experienced in children. Materials \& Methods: An online survey was conducted among parents $(n=1,299)$ of children (4 to 12 years). Latent class analysis (LCA) was performed to identify parental subgroups for children's sunburn, sun exposure and several sun protection behaviours (i.e. applying sunscreen, clothing, seeking shade) in two sun exposure settings (i.e. planned versus incidental). LCA results were validated by assessing predictions of class membership through several sociodemographic characteristics. Results: Reported sunburn in the previous year was frequent $(>40 \%)$. Four latent classes of sunburn-exposureprotection were identified. Overall, the majority of parents reported fair sun protection behaviours. While a low level of protection behaviour was not strongly reflected in lower sunburn rates among the classes, a high level of planned exposure (e.g. going to the beach) seemed to correspond with higher sunburn risk. Parents of younger children and those with more sensitive skin reported sun protection measures more frequently. Older children and those with more sensitive skin experienced more sunburn. Conclusion: This study contributes to current insight into children's sunburn, based on parent-proxy reports. Although a clear differentiation in sunburn risk was not found, several variables, relevant for future interventions, were indicated. By further understanding the complexity of children's sunburn, further research may be directed accordingly.
\end{abstract}

Key words: children's sun safety, parental behaviour, sun exposure, sun protection behaviours sunburn
$\mathrm{M}$ elanoma and non-melanoma skin cancers (NMSC) are some of the most rapidly increasing malignancies worldwide, affecting especially fair-skinned populations [1]. Since the late 1980s, skin cancer incidence has tripled in the United States [2] and nearly quadrupled in the Netherlands [3,4]. Age-standardized incidence rates were estimated at 12.7 for the United States, compared to 25.7 for the Netherlands, 27.6 for Denmark, and 33.6 for Australia per 100,000 cases, respectively, in 2018 [5]. Globally, melanoma represents the most fatal form of skin cancer and its incidence has risen more rapidly than any other malignancy in the last 50 years [2].

Over-exposure to ultraviolet radiation (UVR) and sunburn are major risk factors for developing melanoma, particularly when acquired during childhood [6]. A metaanalysis revealed that having experienced sunburn at least once during childhood almost doubles the risk of developing melanoma [7]. Additionally, although UVR exposure throughout life increases melanoma risk, children's skin is more susceptible to damage, and the harmful effects can latently persist until adulthood [6]. Children's skin has lower levels of protective melanin, resulting in a deeper penetration of UVR and greater damage to the skin [8]. Limiting the amount of UVR and reducing sunburn incidence during childhood is therefore especially important. However, sunburn prevalence among children is high, with reported percentages of children having experienced sunburn at least once during the previous year ranging from $29 \%$ to over $60 \%$ in the Netherlands, United States, Denmark and Switzerland [9-12]. Factors associated with increased sunburn risk among children, such as older age, sun-sensitive skin $[9,13]$, and previous sunburn of parents or children [12-14], were previously reported.

Preventing sunburn among children is of utmost importance. To protect the skin and prevent sunburn in children, several precautions can be taken, such as seeking shade, wearing UV-protective clothing and applying sunscreen. Performing these behaviours simultaneously is recommended, and should start in early life [15]. Sunscreen is undoubtedly the measure applied most frequently [16-18] and is often used as the sole prevention method among parents $[17,19]$. Seeking shade and wearing protective clothing 
are also considered highly important in order to enhance sun safety [20], but sunscreen is still advised to be applied as an adjunct to other forms of sun protection [15].

Multiple approaches are possible in order to promote children's sun safety. Targeting children directly via schools or day-care centres has shown some promising results $[16,21,64]$. However, since parents and caregivers play a vital role in establishing children's sun safety and subsequent prevention of sunburn experience, this indirect approach is also considered very important $[14,16,22]$. Parents are primarily responsible for the application of sun protection measures for their children [17] and are most important in teaching children to perform their own sun protection behaviours [14]. The significant influence of parents, as active agents and role models, is well reflected in strong correlations between parents' own sun protection behaviour and their children's sun safety [12, 23-25]. Parents are therefore recommended to strictly adhere to sun protection guidelines and to teach their children adequate sun protection behaviours $[15,21]$, rendering them an essential target group for communication and intervention aimed at preventing children's sunburn.

Despite various studies reporting on parent-for-child sun protection behaviours, the association between the extent of UVR exposure in children and parent-for-child sun protection behaviours is under-explored. Equally unknown is how this exposure-protection relationship affects children's sunburn experiences. Previous studies have focused on very young children $[24,26,27]$, or did not report sun exposure patterns $[28,29]$. The link between sun exposure, sun protection behaviours and sunburn is regarded as complex and needs further clarification. Hence, in order to gain insight into children's sunburn, identification of risk patterns associated with UVR exposure and parent-for-child sun protection behaviours in various situations is necessary. The extent to which sun protection behaviours are necessary depends on the activities in which sun exposure is acquired. For example, when not actively focused on spending time outdoors, the level of effort to protect ones' skin may be lower than that of those who intentionally expose themselves to the sun $[19,30]$. Consequently, the risk of sunburn increases in these unintentional sun exposure situations (e.g. playing outdoors) due to limited shade availability, prolonged duration of exposure, or reliance on sunscreen use only, as shown by a recent study among adolescents and adults in New Zealand [31] and Denmark [32]. Two German studies among parents of very young children (three to six years) revealed different parent-for-child sun protection behaviours; shade-seeking behaviour and wearing hats and sunglasses were applied more often in a beach setting than during everyday outdoor activities [33, 34]. Moreover, parental sun protection behaviours among older children vary across sun exposure situations [18, 35], and their relationship with sunburn is largely undocumented. There is evidence that the more intense and intermittent the UVR, e.g. during recreational activities, the greater the risk of melanoma $[6,8]$. Therefore, shedding light on the association between different types of sun exposure, sun protection strategies and their relation to children's sunburn is expected to generate a better understanding of sunburn risk in different exposure settings. This knowledge would facilitate the development and implementation of targeted policies and interventions to counteract the increasing melanoma incidence rates.
This study aimed to explore the heterogeneity of interrelations between: (1) children's sun exposure; (2) parental sun protection behaviours; and (3) children's sunburn frequency. To achieve this goal, we applied latent class analysis (LCA), a model-based clustering technique [36], to empirically derive typologies capturing distinct patterns of inter-connections across the three domains. Furthermore, links between the identified classes and parental and children's socio-demographic characteristics were investigated to explore possible subgroups that deserve specific attention in future interventions.

\section{Materials and methods}

\section{Procedure and participants}

This study was part of a longitudinal survey study, of which the baseline data (June 2016 [T0]) was analysed. The study was exempt from medical ethical evaluation since participants were not exposed to medical procedures [37]. The collected data is not traceable to specific participants in the dataset [38].

Eligible participants, caring for at least one child of primary school age (4 to 12 years), were retrieved from an existing study panel and received one invitation and one reminder email. A sample of 2,449 parents, representative of the Dutch general population according to education level and income, were approached by the research organisation TNS-KANTAR. Informed consent was acquired online [39]. In total, 1,299 Dutch parents completed the online questionnaire. Parents obtained gift vouchers after completing the survey.

\section{Measurement}

The online questionnaire assessed, amongst others: (1) demographic characteristics; (2) children's sunburn experiences; (3) the extent of children's sun exposure; and (4) parental sun protection behaviours (i.e. sunscreen use, clothing, and seeking shade) in both planned and incidental sun exposure situations. Planned situations concerned sun exposure generally anticipated by the parent and/or the child (e.g. going to the beach or swimming pool), whereas incidental situations consisted of less intentional sun exposure situations (e.g. when walking, cycling or playing outdoors). Children's sunburn, sun exposure and sun protection behaviours during the previous summer season were assessed for the youngest child in the parents' household. The full questionnaire can be retrieved from Open Science Framework (OSF): https://osf.io/zncwq/. This study is reported in accordance with STROBE guidelines for observational research [40].

\section{Socio-demographic characteristics}

Parental and children's age, sex, skin type and parental educational level and province of residence were assessed. Parental age was categorized in three groups (18-34 years, $35-44$ years, and $\geq 45$ years), as was children's age (4 to 6 years, 7 to 9 years, and $\geq 10$ years), the latter according to the Dutch primary school system. Age categories were referred to as young, middle-aged and old. Parents' 
and children's skin type were categorized as: (I) burns very quickly, tans never or rarely; (II) burns quickly, tans slowly; (III) burns rarely, tans easily; and (IV) burns almost never, tans very easily. Educational level was classified as (1) low, (2) intermediate and (3) high education, cf. guidelines of Statistics Netherlands [41]. The 12 Dutch provinces of residence were subdivided into (1) North, (2) East, (3) West and (4) South, cf. classification of Dutch Ministry of the Interior and Kingdom Relations [42].

\section{Children's sunburn}

The frequency of children's sunburn was assessed using one item, in which parents were asked how often their child had experienced sunburn in the past 12 months. A 5-point Likert scale was used $(1=$ never; $2=1-2$ times; $3=3-4$ times; $4=4$ 5 times; and $5=>5$ times).

\section{Children's sun exposure}

The amount of received sun exposure was assessed by asking parents the frequency with which their child was outside on sunny days during the previous 12 months, using a 5 -point Likert scale $(1=$ never; $2=$ rarely; $3=$ sometimes; $4=$ often; $5=$ as often as possible). The questions regarding sun exposure were asked using two items; one regarding planned situations and one for incidental situations. Planned sun exposure involved intentional sun-seeking behaviour (e.g. going to the beach or swimming pool) and incidental sun exposure consisted of all other situations in which children were sun-exposed (e.g. playing outdoors, cycling). Parents received an explanatory text regarding how to distinguish between these sun exposure situations.

\section{Parental sun protection behaviours}

Three parent-for-child sun protection behaviours were assessed: (1) sunscreen use; (2) clothing; and (3) seeking shade. The occurrence of these behaviours was questioned regarding the two (planned and incidental) sun exposure situations, resulting in six items. A 5-point Likert scale was again used to assess the frequency of sun protection behaviours $(1=$ never; 2 = rarely; $3=$ sometimes; $4=$ often; and $5=$ always). Sufficient sunscreen application encompassed using an $\mathrm{SPF} \geq 30$, applying sunscreen 30 minutes prior to sun exposure, and reapplying sunscreen every two hours. For clothing, wearing a T-shirt that covers the shoulders, a hat and sunglasses was regarded as sufficient. Lastly, seeking shade between UV peak hours, 12 and 3 PM, was considered adequate. Explanatory text regarding sufficient sun protection behaviour was provided in the questionnaire (https://osf.io/zncwq/).

\section{Analysis of variables}

Data for statistical analyses was prepared by recoding the categorical variables. An inspection of the frequency distribution of the answers showed a very low count in some response categories. For this reason, all categorical variables were collapsed to avoid sparsity problems. Children's sunburn frequency was recoded as never (1), 1-2 times (2), and $>3$ times (3), while children's sun exposure in both planned and incidental situations was recoded as never (1), rarely and sometimes (2), and often and as often as possible (3), and parental execution of sun protection behaviours in both sun exposure situations as never (1), rarely and sometimes (2), and often and always (3). Based on this recoding, three answer categories were obtained across all items. First, the category with the least protective answers included the responses with $>$ three episodes of sunburn, the highest reported levels of sun exposure and the lowest reported levels of sun protection behaviours. Second, the intermediate answer category included responses with 1-2 episodes of sunburn, being rarely and occasionally exposed to the sun, and rarely and occasionally demonstrating sun protection behaviours. Lastly, the category with the most protective answers included no previous episodes of sunburn, the lowest reported level of sun exposure and highest levels of reported sun protection behaviours.

\section{Statistical methods}

Descriptive statistics (means, standard deviations and percentages) were computed to examine the distribution of participants' characteristics, children's sunburn frequency, children's sun exposure and the frequency of parental sun protection behaviours. McNemar-Bowker and Chi-square tests were conducted to explore differences in sample protection behaviours in planned vs. incidental sun exposure situations and for sunburn frequency, respectively. Significance level was set at 5\%. IBM SPSS Statistics for Windows, version 25.0, was used [43].

\section{Latent class analysis-model fitting}

Latent class analysis (LCA), a model-based clustering technique, was conducted to explore the heterogeneity of children's sunburn and behavioural patterns (both children's sun exposure and parental sun protection behaviours) [36]. The non-directly observable data-driven classes were revealed by grouping participants with similar responses to items related to sunburn, sun exposure, and sun protection behaviours, but different from other groups. Class enumeration, i.e. determining the number of latent classes, was guided by the model fit Akaike Information Criterion (AIC) and adjusted Bayesian Information Criterion (BIC) [44], while visually assessing whether extracted classes were qualitatively distinct from each other. After settling for the final number of classes, post-hoc posterior probabilities of class membership was computed for each subject [36], who was then assigned to the class with the highest posterior probability. The index of entropy, though not used to guide class enumeration, was also reported to indicate the quality of classification uncertainty (entropy values above 0.80 are considered to be of high classification value). LCA was performed using SAS software version 9.4 [45].

\section{Class profiling}

Chi-square tests were performed to explore unadjusted differences among extracted classes for socio-demographic characteristics (parental age, sex, skin type, province of residence and educational level, and children's age, sex and skin type). A multinominal linear regression model was fitted to establish which of these characteristics remained independently linked to class membership after mutual adjustment. Estimated odds ratio (OR) and $95 \%$ confidence intervals (CIs) are displayed in forest plots. To avoid multicollinearity in the multinomial logistic regression, age and skin type of the child rather than of the parents were included in the model. 
Table 1. Parent-for-child sun protection behaviours $(n=1299)$.

\begin{tabular}{|c|c|c|c|c|c|}
\hline \multicolumn{4}{|c|}{ Comparisons between planned and incidental exposure } & \multirow{2}{*}{$\begin{array}{l}\text { McNemar-Bowker test } \\
\chi^{2}(3)=330.91\end{array}$} & \multirow{2}{*}{$\begin{array}{l}\boldsymbol{p} \text {-value } \\
<0.001\end{array}$} \\
\hline \multirow{4}{*}{ Sunscreen } & & Planned & Incidental & & \\
\hline & Never & $6(.5)$ & $16(1.2)$ & & \\
\hline & Rarely/Sometimes & $98(7.5)$ & $431(33.2)$ & & \\
\hline & Often/Always & $1195(92.0)$ & $852(56.6)$ & & \\
\hline \multirow{4}{*}{ Clothing } & & Planned & Incidental & $\chi^{2}(3)=28.01$ & $<0.001$ \\
\hline & Never & $23(1.8)$ & $24(1.8)$ & & \\
\hline & Rarely/Sometimes & $643(49.5)$ & $551(42.4)$ & & \\
\hline & Often/Always & $633(48.7)$ & $724(55.7)$ & & \\
\hline \multirow{4}{*}{ Seeking shade } & & Planned & Incidental & $\chi^{2}(3)=18.32$ & $<0.001$ \\
\hline & Never & $53(4.1)$ & $59(4.5)$ & & \\
\hline & Rarely/Sometimes & $731(56.3)$ & $782(60.2)$ & & \\
\hline & Often/Always & $515(39.6)$ & $458(35.3)$ & & \\
\hline \multicolumn{6}{|c|}{ Parent-for-child sun protection behaviours stratified by child's age and skin type (Chi-square) } \\
\hline & & Child's age & p-value & Child's skin type & $p$-value \\
\hline \multirow[t]{2}{*}{ Sunscreen } & Planned & $x^{2}(4)=6.57$ & .16 & $\chi^{2}(6)=16.55$ & 0.01 \\
\hline & Incidental & $\chi^{2}(4)=15.32$ & .004 & $\chi^{2}(6)=28.56$ & $<0.001$ \\
\hline \multirow[t]{2}{*}{ Clothing } & Planned & $\chi^{2}(4)=13.21$ & .01 & $\chi^{2}(6)=36.35$ & $<0.001$ \\
\hline & Incidental & $\chi^{2}(4)=26.66$ & $<.001$ & $\chi^{2}(6)=24.20$ & $<0.001$ \\
\hline \multirow[t]{2}{*}{ Seeking shade } & Planned & $\chi^{2}(4)=17.72$ & .001 & $\chi^{2}(6)=11.18$ & 0.08 \\
\hline & Incidental & $\chi^{2}(4)=23.63$ & $<.001$ & $\chi^{2}(6)=16.12$ & 0.013 \\
\hline
\end{tabular}

\section{Results}

\section{Socio-demographic characteristics}

Of the included parents, 773 (59.5\%) were mothers and the questionnaire was answered by $656(50.5 \%)$ boys. Parents' age was normally distributed (range: 18 to 69 years) and most often between 40 and 44 years old (32.6\%). Children's age was not evenly distributed, and most often between four and six years old $(n=595 ; 45.8 \%)$. Most children had skin type III (burns rarely) (49.7\%), followed by skin type II (burns quickly) (35.9\%). Most parents had received higher education $(n=625 ; 48.3 \%)$.

\section{Sunburn, sun exposure and sun protection behaviour}

Overall, during the previous year, 550 children $(42.4 \%)$ had experienced at least one episode of sunburn. Older children $\left(\chi^{2}(4)=12.80 ; p=0.012\right)$ and children with more sensitive skin $\left(\chi^{2}(6)=111.52 ; p<0.001\right)$ experienced more sunburn. Parents of younger children and children with more sensitive skin reported more frequent execution of almost all sun protection behaviours, compared to parents of younger children and with less sensitive skin types. Sunscreen use
Table 2. Model fit evaluation of latent classes.

\begin{tabular}{|llll|}
\hline $\begin{array}{l}\text { Number of } \\
\text { latent class }\end{array}$ & AIC & Adjusted BIC & Entropy \\
\hline 3 & 931.18 & 1042.78 & 0.88 \\
\hline 4 & $\mathbf{8 9 1 . 2 1}$ & $\mathbf{1 0 4 0 . 6 7}$ & $\mathbf{0 . 8 2}$ \\
\hline 5 & 862.12 & 1049.45 & 0.84 \\
\hline 6 & 845.25 & 1070.44 & 0.85 \\
\hline
\end{tabular}

and seeking shade were more often practised in planned situations, and clothing in incidental situations. Table 1 provides an overall summary of the whole sample, and as such, only a general overview of the marginal distributions. A more refined glimpse into the data is provided by the LCA findings.

\section{Categorisation of subgroups (latent class analyses)}

Models with three to six latent classes were fitted. Table 2 shows model fit assessment criteria that were used to guide class enumeration. These were not in full agreement, but visual inspection of the distinctiveness of the classes 


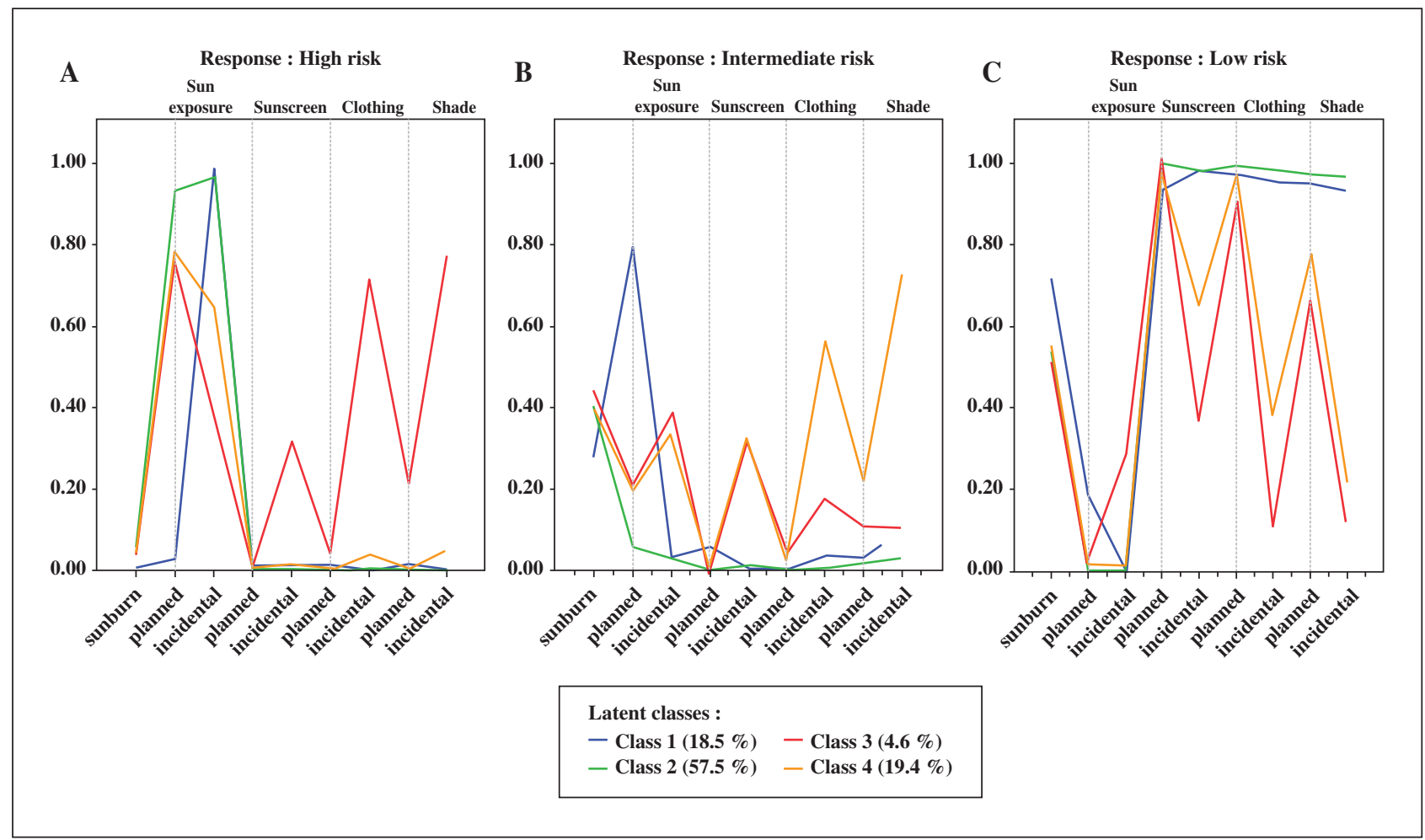

Figure 1. Profile plot with four latent classes depicting the probability (per class) of selecting the high (A), intermediate (B) and low (C) risk answer category for sunburn, sun exposure and sun protection questions. The prevalence of classes is presented as $\%$.

together with the BIC indicated that a solution with four classes was a good fit to the data.

Figure 1 illustrates the distinct response patterns to the sunburn, exposure and protective behaviour questions of the four extracted classes. The plot shows the probability of class members to report high-risk, intermediate-risk and low-risk answers to sunburn, sun exposure and sun protection items. For instance, the $\mathrm{y}$-axis of subplot 1A shows the probability for the high-risk answer category, i.e. highest frequency of sunburns ( $\leq$ three episodes of sunburn), greatest sun exposure (often and as often as possible) and lowest reported levels of sun protection behaviours (never performed), while the $\mathrm{x}$-axis indicates whether the situation was planned or incidental. Similarly, figure $1 B, C$ shows the probability for intermediate- and low-risk answer patterns. Sunburn probability was relatively high (between $\sim 40$ and $45 \%$ for one or two cases of sunburn; $\sim 50 \%$ for no sunburn) for three out of the four classes (1B), showing low discriminatory power between these classes for this item. Class 1 demonstrated a lower sunburn risk ( $\sim 30 \%$ of cases for one or two episodes of sunburn; $\sim 70 \%$ for no sunburn). The probability of children with $\leq$ three reported episodes of sunburn was very low among all classes (1A).

Sun exposure patterns showed a larger discriminatory power between classes. Individuals of Class 1 and 2, which made up the majority of parents $(>70 \%)$, reported the highest levels of exposure in incidental situations, and the remaining classes ( 3 and 4 ) reported lower incidental exposure relative to planned exposure (1A). Of note, Class 1 showed almost complete withholding of planned sun exposure.
Sun protection patterns were again distinct between the classes. Class 1 and 2 reported relatively high levels of sun protection behaviours in both situations, whereas the remaining two classes ( 3 and 4 ) showed more variability, with protective behaviours being more frequent in planned rather than incidental situations. The latter classes had a clear preference for sunscreen use, followed by clothing and seeking shade, respectively. Of note, these classes differed in the degree of sun protection behaviours, with Class 3 showing the least sun protection behaviour (figure 1C).

The four classes were named primarily based on parental sun protection behaviour:

Class 1 with adequate sun protection behaviour $(n=288$; $30.6 \% \geq$ one sunburn) was considered the best performing class, as reflected by: a) the lowest probability of sunburn; b) consistent reporting of high levels of sun protection behaviour; and c) no exposure in planned situations and high exposure in incidental situations.

Class 2 with adequate sun protection behaviour $(n=726$; $46.3 \% \geq$ one sunburn) was defined as the second-best performing class, as reflected by: a) moderate probability of sunburn; b) consistent reporting of high levels of sun protection behaviour; and c) high levels of sun exposure in planned and incidental situations, indicating unfavourable exposure patterns.

Class 3 with inadequate sun protection behaviour $(n=53$; $51 \% \geq$ one sunburn) was considered the worst performing class, as reflected by: a) moderate probability of sunburn; b) low levels of sun protection behaviours, in particular, in incidental situations; and c) lower levels of sun exposure 
Table 3. Socio-demographic characteristics and class profiling.

\begin{tabular}{|c|c|c|c|c|c|}
\hline & $\begin{array}{l}\text { Best class } \\
(n=288 \\
18.5 \%) \\
n(\%)\end{array}$ & $\begin{array}{l}\text { Second best class } \\
(n=726 ; 57.5 \%) \\
n(\%)\end{array}$ & $\begin{array}{l}\text { Worst class } \\
(n=53 ; 4.6 \%) \\
n(\%)\end{array}$ & $\begin{array}{l}\text { Intermediate class } \\
(n=232 ; 19.4 \%) \\
n(\%)\end{array}$ & $\begin{array}{l}p \text { value } \\
\text { (Chi-Square) }\end{array}$ \\
\hline \multicolumn{6}{|l|}{ Parents' characteristics } \\
\hline \multicolumn{6}{|l|}{ Age } \\
\hline Young & $53(18.4)$ & $102(14.0)$ & $6(11.3)$ & $21(9.1)$ & \multirow[t]{3}{*}{0.003} \\
\hline Middle-aged & $182(63.2)$ & $449(61.8)$ & $27(50.9)$ & 143 (61.6) & \\
\hline Old & $53(18.4)$ & $175(24.1)$ & $20(37.7)$ & $68(29.3)$ & \\
\hline \multicolumn{6}{|l|}{ Sex } \\
\hline Male & $110(38.2)$ & $286(39.4)$ & $26(49.1)$ & $104(44.8)$ & \multirow[t]{2}{*}{0.220} \\
\hline Female & $178(61.8)$ & $440(60.6)$ & $27(50.9)$ & $128(55.2)$ & \\
\hline \multicolumn{6}{|l|}{ Skin type } \\
\hline I: burns very quickly & $31(10.8)$ & $77(10.6)$ & $2(3.8)$ & $9(3.9)$ & \multirow[t]{4}{*}{$<0.001$} \\
\hline II: burns quickly & $124(43.1)$ & $284(39.1)$ & $12(22.6)$ & $96(41.4)$ & \\
\hline III: burns rarely & $114(39.6)$ & $322(44.4)$ & $30(56.6)$ & $101(43.5)$ & \\
\hline IV: burns almost never & $19(6.6)$ & $43(5.9)$ & $9(17.0)$ & $26(11.2)$ & \\
\hline \multicolumn{6}{|l|}{ Education } \\
\hline Low & $36(12.5)$ & $102(14.0)$ & $11(20.8)$ & $43(18.5)$ & \multirow[t]{3}{*}{0.118} \\
\hline Intermediate & $111(38.5)$ & $278(38.5)$ & $20(37.7)$ & $69(29.7)$ & \\
\hline High & $141(49.0)$ & $343(47.2)$ & $21(39.6)$ & $120(51.7)$ & \\
\hline \multicolumn{6}{|l|}{ Province } \\
\hline North & $19(6.6)$ & $68(9.4)$ & $2(3.8)$ & $19(8.2)$ & \multirow[t]{4}{*}{0.135} \\
\hline East & $74(25.7)$ & $162(22.3)$ & $8(15.1)$ & $61(26.3)$ & \\
\hline South & $132(45.8)$ & $308(42.4)$ & $33(62.3)$ & $100(43.1)$ & \\
\hline West & $63(21.9)$ & $187(25.8)$ & $10(18.9)$ & $52(22.4)$ & \\
\hline \multicolumn{6}{|c|}{ Children's characteristics } \\
\hline \multicolumn{6}{|l|}{ Age } \\
\hline Young & $172(59.7)$ & $326(44.9)$ & $15(28.3)$ & $82(35.3)$ & \multirow[t]{3}{*}{$<0.001$} \\
\hline Middle-aged & $71(24.7)$ & $221(30.4)$ & $22(41.5)$ & $71(30.6)$ & \\
\hline Old & $45(15.6)$ & $179(24.7)$ & $16(30.2)$ & $79(34.1)$ & \\
\hline \multicolumn{6}{|l|}{ Sex } \\
\hline Boy & $145(50.3)$ & $366(50.4)$ & $25(47.2)$ & $120(51.7)$ & \multirow[t]{2}{*}{0.945} \\
\hline Girl & $143(49.7)$ & $360(49.6)$ & $28(52.8)$ & $112(48.3)$ & \\
\hline \multicolumn{6}{|l|}{ Skin type } \\
\hline I: burns very quickly & $24(8.3)$ & $51(7.0)$ & $1(1.9)$ & $5(2.2)$ & \multirow[t]{4}{*}{0.001} \\
\hline II: burns quickly & $112(38.9)$ & $264(36.4)$ & $14(26.4)$ & $76(32.8)$ & \\
\hline III: burns rarely & $131(45.5)$ & $363(50.0)$ & $29(54.7)$ & $123(53.0)$ & \\
\hline IV: burns almost never & $21(7.3)$ & $48(6.6)$ & $9(17.0)$ & $28(12.1)$ & \\
\hline
\end{tabular}

in planned and especially incidental situations, indicating a more favourable exposure pattern.

Class 4 with inadequate sun protection behaviour $(n=232$; $42.7 \% \geq$ one sunburn) was defined as the intermediate performing class, as reflected by: a) moderate probability of sunburn; b) lower levels of sun protection behaviours; and c) lower levels of sun exposure in planned and especially incidental situations.

\section{Socio-demographic class profiling}

Next, we compared whether parents' and children's characteristics differed across the four classes. In general, the majority of parents were middle-aged, had skin that burns quickly (II) or rarely (III), were highly educated and lived in the western part of the Netherlands. The majority of children also had skin type II or III. Differences in both parents' and children's age and skin type (table 3) distributions were observed across classes, with the best and second-best class including younger children with more sensitive skin types.
These differences were further confirmed after mutual adjustment (figure 2). Results revealed that parents assigned to the best two classes had the youngest children with most sensitive skin complexions, while the best group (Class 1) included the youngest children. The two remaining classes included parents of older children, with less sensitive skin types. The total model was adjusted for parental age, sex, skin type, province of residence, educational level and children's age, sex and skin type. All model parameter estimates can be retrieved from Appendix I.

\section{Discussion}

This study exposes differences in parental sun protection, children's sun exposure patterns and children's sunburn. Overall, sunburn frequency was high $(>40 \%)$ among all children, and parents reported fair sun protection behaviour. LCA revealed four sun typologies 


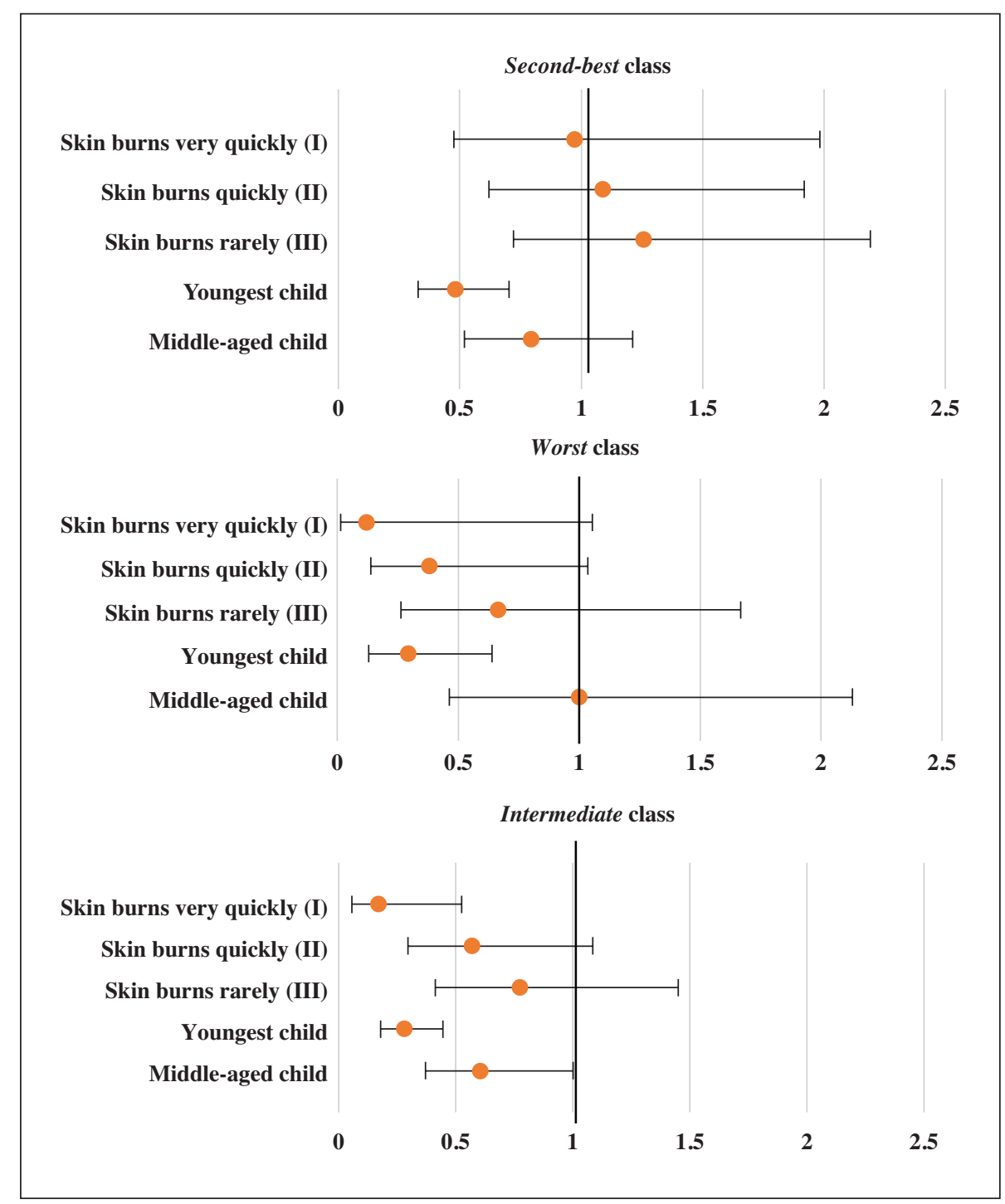

Figure 2. Forest plots with odds ratios (ORs) and associated Confidence Intervals (CIs) (95\%) of statistically significant results of the multinominal (multivariable) logistic regression, demonstrating socio-demographic characteristics linked to class membership. OR are provided with respect to the best class (1). Reference group for skin type is 'Skin never burns (IV)'; Reference group for children's age is 'oldest child' (see also supplementary material).

(burn-exposure-protection), which, although differed with regards to frequency and type of sun protective strategy, could not be firmly distinguished in terms of sunburn frequency for three of the four classes. Parents engaged in more protective measures during planned rather than incidental sun exposure. Avoiding sun exposure in planned situations (e.g. going to the beach or swimming pool) seemed to be associated with a lower sunburn risk. These findings seem to confirm the complexity of the relationship between children's sunburn, sun exposure and parental sun protection behaviours; although the majority of parents reported relatively high levels of sun protection measures (Class 1 and 2 ), this resulted in a lower probability of sunburn among only a small group of children (Class 1). This latter group included parents of young children and children with more sensitive skin types, who were inclined to limit sun exposure for their children in planned situations.
Substantial numbers of children in all four classes experienced at least one case of sunburn in the previous year, which is comparable to findings in other countries $[9,10,12]$. Parents in the best class with lowest, yet notable, sunburn prevalence $(>30 \%)$ were engaging in all sun protection behaviours while limiting their children's sun exposure in planned situations. They seemed to have been highly aware of the importance of sun protection behaviours and sun avoidance, but their children's sun exposure in incidental situations was still high. This may indicate, in line with previous research, that parents underestimate the risk of UVR exposure during everyday activities [34, 46], or that they are not well prepared for sun protection in these settings [47]. Parental awareness about sun exposure risk in multiple settings is therefore highly important in order to minimize sunburn risk, even among parents reporting high levels of sun protection behaviours. 
High levels of sun protection behaviour were observed with the majority of parents frequently reporting seeking shade and dressing appropriately, in line with sun safety recommendations. This finding contrasts with earlier studies using comparable measures $[10,17,18,48]$. The observed discrepancy between a high level of reported sun protection behaviour and substantial sunburn risk is noteworthy. This may indicate that, while parents may believe they are adhering to sun safety recommendations, the practice of these recommendations is suboptimal. Several studies reported on partial sun protection measures for children. For example, sunscreen efficacy mainly depends on the way in which it is applied [49]. However, sufficient sunscreen application does not appear to be achieved by parents [47], as indicated by their failure to apply sunscreen 30 minutes before going outdoors [48], skipping sensitive body parts [19], applying insufficient amounts of sunscreen [50] or not reapplying every two hours [9]. Parents' erroneous perception that they have sufficiently protected their child with sunscreen may lead them to prolong their time spent in the sun, resulting in sunburn $[9,51]$. Furthermore, studies reported that children are less likely to be protected by sunglasses and a hat than by a T-shirt $[52,53]$, while concurrent use is recommended [15]. Hence, it is possible that the parents in the current study over-estimated their adherence to specific sun protection recommendations, despite the information provided regarding sufficient sun protection in the questionnaire. Another explanation for this finding could be that parents were unaware of their children's skin sensitivity or that they underestimated previous sunburn experiences $[14,27,47]$.

Although research on sun exposure patterns in children is scarce, recent studies from Germany reveal that the amount of time very young children (aged three to six) spend outside during peak UV hours in various settings was high [33], and that older children receive high doses of UVR in playgrounds [54]. Our findings also suggest high levels of exposure, in particular, among young children in both sun exposure situations. The parents of young children reported adequate sun protection behaviour when their children were highly exposed in planned situations, but this was not echoed in children with low sunburn risk. A lower sunburn risk was, however, found among young children whose parents limited their sun exposure in planned situations. These findings emphasize the difficulty in optimising sun protection in planned sun exposure situations and may lead to the question of whether sunburn prevention is entirely possible or realistic in such situations. Protecting children sufficiently on the beach or by the swimming pool is particularly challenging as the availability of shaded areas is often lacking, sunscreen has to be reapplied more frequently, and clothing may be perceived as a burden $[47,53]$.

Parents assigned to the best-performing class had the youngest children with more sensitive skin. The greater protection for younger children is in agreement with previous research [13, 18, 33]. Since sun protection attitudes and behaviours decrease during adolescence and sunburn prevalence increases, establishing sun safety for older children is considered highly important [10, 17, 29]. Increased parental vigilance and sun protection behaviours among children with lighter skin types has also been described in earlier work [27, 28, 34, 47]. Similarly, parents with darker skin complexions may assume that their skin phenotype protects them from UVR [26], resulting in less sufficient sun protection for their children [48]. The association between children's sun-sensitivity and better sun protection and thus lower sunburn rates might seem obvious, but is often not apparent $[9,10,25]$. In the present study, a comparable relation between more sensitive skin types and high parental sun protection behaviours was found. Despite this, these children still seemed to be at high risk of sunburn. The association between children's skin sensitivity, sun protection behaviours and their risk of sunburn, however, deserves closer examination in order to establish future sun safety interventions.

\section{Strengths and limitations}

This study has several strengths that are worth mentioning. Firstly, the assessment of comprehensive sun protection behaviours in distinctive sun-exposing situations provides a novel and insightful approach compared to existing parental sun protection research. Secondly, by using a large sample size and including more than five indicators, the validity of latent class fitting was optimised.

The task of exploring associations between sunburn, sun exposure and sun protection behaviours is not straightforward and is hampered by certain methodological challenges. Firstly, although sunburn rates in our study sample were comparable or even higher than those in the literature, the practice of parental sun protection behaviours was high. A bias due to social pressure could have been at play, urging parents to report more frequent protection behaviour. Furthermore, since all questions were asked regarding the previous year, recall bias may have limited the accuracy of the self-reported behaviours [55]. Despite our efforts to reduce these biases by stressing confidentiality and non-disclosure of the study, these biases should be considered when interpreting the present findings. Secondly, sun exposure was broadly defined in this study, and details such as weather conditions, exact time of day and duration of activities were not taken into account. Self-reported sun protection has been shown to be a valid measure when contextual factors, such as time and activity, are specifically assessed [56], or when the retrospective timeframe is as short as possible [57]. Future assessment of sun exposure should ideally include tailored questions regarding specified sun-exposing situations. Lastly, the findings in this study may not readily be generalised to Dutch parents since the majority of parents received a high level of education and most children were aged between four and six years.

\section{Implications}

The majority of parents seemed to practice recommended sun protection frequently, but variability in sun protection behaviours and sun exposure patterns was observed. Furthermore, specific individual characteristics, such as skin type and children's age, may in part account for this variability. Despite the emergence of a positive picture of sun protection behaviours, a high sunburn risk was still prevalent among the majority of children. These exploratory findings hint at directions for further research in order to investigate children's sunburn in more detail.

First, all indicators were assessed subjectively. For future efforts, we recommend the application of more objective 
measurements of sunburn and UVR exposure as well as sun protection behaviours. For example, some parents have difficulty recognising sunburn as such, e.g. being unaware that reddening of the skin already signifies sunburn, leading to an under-estimation of sunburn prevalence [47]. Objectively assessing sunburn and its severity would be worthwhile to improve risk-profiling (e.g. through biometric measures of melanin and erythema on the skin [58]), since sunburn and particularly severe and painful sunburn are highly correlated with melanoma development in later life [7]. Furthermore, although validation studies show fair to strong correlations between self-reported and observed exposure [57, 59], collecting objective data about children's UVR exposure has been strongly advocated [60]. Environmental effects such as shade coverage, altitude or humidity cannot be measured in self-reported questionnaires [61]. Because detailed information on actual received UVR dosage is lacking among children, the formulation of specific policy and guidelines, weighted for specific environmental characteristics (e.g. latitude, cloudiness, seasonality), cannot be made. As stated, parents may feel obliged to report desirable sun protection behaviours, or may over-estimate their behaviours, which can both affect the validity of the findings. Investigating the practice of specific sun protection measures (e.g. using $\geq$ SPF 30, reapplication patterns [49] and wearing sunglasses as well as a hat) could provide further insight into parental sun protection behaviours.

Second, since the findings imply that planned exposure (e.g. at the beach or swimming pool), in particular, may increase the risk of sunburn, implementing health-promoting environmental cues in these settings are advisable $[62,63]$. For instance, by providing shade, promotion of UV-protective clothing and freely available sunscreen, both parents and children can be encouraged to practice sun protection behaviours more effectively.

Third, similar to earlier studies [13, 18, 23], the present study reveals that older children seem to receive less protective behaviours from their parents, and experience more sunburn than younger children. Future interventions should target older children, especially before entering the adolescent phase $[25,29]$.

\section{Conclusions}

This study emphasizes the complexity of the association between children's sunburn, their sun exposure and parental sun protection behaviours, while indicating directions for further investigation. The findings indicate that despite fair to good reported sun protection behaviours, sunburn occurrence is still high among children. Furthermore, the greatest sun protection was reported for younger children with more sensitive skin types, which was reflected in a lower sunburn risk among a small group of children. We were unable to fully unravel the underlying mechanisms of the associations between sun exposure, sun protection behaviours and sunburn. Therefore, recommendations for increasing sun safety in children are provided with a cautionary note. Further research is required to clarify these complex relationships in order to improve or develop tailored interventions.
Disclosures. Financial support: none. Conflicts of interest: none.

\section{Supplementary data}

Supplementary data associated with this article can be found, in the online version, at doi:10.1684/ejd.2021.4100.

Table S1: 11 model parameter estimates of multinominal linear regressions.

\section{References}

1. Lai V, Cranwell W, Sinclair R. Epidemiology of skin cancer in the mature patient. Clin Dermatol 2018;36(2): 167-76.

2. Karimkhani $C$, Green AC, Niisten $T$, et al. The global burden of melanoma: results from the Global Burden of Disease Study 2015. Br J Dermatol 2017; 177(1): 134-40.

3. Slaper $H$, Van Dijk A, Den Outer $P$, van Kranen $H$, Slobbe L. UV-straling en gezondheid: Probleemveld en kennisbasis bij het RIVM. Bilthoven: Rijksinstituut voor Volksgezondheid en Milieu (RIVM), 2017. Available at: https://www.rivm.nl/bibliotheek/rapporten/20170074.pdf (accessed 02 Sep 2020).

4. Hollestein L, de Vries E, Nijsten T. Trends of cutaneous squamous cell carcinoma in the Netherlands: increased incidence rates, but stable relative survival and mortality 1989-2008. Eur J Cancer 2012; 48(13): 2046-53.

5. Khazaei Z, Ghorat F, Jarrahi A, Adineh $H$, Sohrabivafa $M$, Goodarzi E. Global incidence and mortality of skin cancer by histological subtype and its relationship with the human development index (HDI); an ecology study in 2018. World Cancer Res J 2019; 6(2): el 3. 6. Gandini S, Sera F, Cattaruzza MS, et al. Meta-analysis of risk factors for cutaneous melanoma: II. Sun exposure. Eur J Cancer 2005; 41 (1): 45-60.

7. Dennis LK, Vanbeek M, Freeman LEB, Smith BJ, Dawson DV, Coughlin JA. Sunburns and risk of cutaneous melanoma: does age matter? A comprehensive meta-analysis. Ann Epidemiol 2008; 18(8): 614-27

8. Cestari T, Buster K. Photoprotection in specific populations: children and people of color. J Am Acad Dermatol 2017;76(3): S1 10-21.

9. Hall HI, McDavid K, Jorgensen CM, Kraft JM. Factors associated with sunburn in white children aged 6 months to 11 years. Am J Prev Med 2001; 20(1): 9-14.

10. Ackermann S, Vuadens A, Levi F, Bulliard J-L. Sun protective behaviour and sunburn prevalence in primary and secondary schoolchildren in western Switzerland. Swiss Med Wkly 2016; 146: w14370

11. Thoonen K, van Osch L, Crutzen R, de Vries H, Schneider $F$. Identification of relevant sociocognitive determinants explaining multiple parental sun protection behaviors. Health Educ Beh $2021 ; 10901981211010434$, Online ahead of print.

12. Behrens $C L$, Thorgaard $C$, Philip $A$, Bentzen J. Sunburn in children and adolescents: associations with parents' behaviour and attitudes. Scand J Public Health 2013; 41 (3): 302-10.

13. Tripp MK, Peterson SK, Prokhorov AV, et al. Correlates of sun protection and sunburn in children of melanoma survivors. Am J Prev Med 2016; 51 (3): e77-85.

14. O'Riordan DL, Geller AC, Brooks DR, Zhang Z, Miller DR. Sunburn reduction through parental role modeling and sunscreen vigilance. J Pediatr 2003; 142(1): 67-72. 
15. Watson M, Garnett E, Guy GP, Holman DM. The surgeon general's call to action to prevent skin cancer. 2014. Available at: https://stacks.cdc.gov/view/cdc/24419 (accessed 04 Aug 2020).

16. Dadlani C, Orlow SJ. Planning for a brighter future: a review of sun protection and barriers to behavioral change in children and adolescents. Dermatol Online J 2008; 14(9): 1.

17. Stanton WR, Janda M, Baade PD, Anderson P. Primary prevention of skin cancer: a review of sun protection in Australia and internationally. Health Promot Int 2004; 19(3): 369-78.

18. Thoonen $K$, Schneider $F$, Candel $M$, de Vries $H$, van Osch L. Childhood sun safety at different ages: relations between parental sun protection behavior towards their child and children's own sun protection behavior. BMC Public Health 2019; 19(1): 1044 .

19. Robinson JK, Rigel DS, Amonette RA. Summertime sun protection used by adults for their children. J Am Acad Dermatol 2000; 42(5): 746-53

20. Linos E, Keiser E, Fu T, Colditz G, Chen S, Tang JY. Hat, shade, long sleeves, or sunscreen? Rethinking US sun protection messages based on their relative effectiveness. Cancer Causes Control 2011;22(7): 1067-71

21. Saraiya M, Glanz K, Briss PA, et al. Interventions to prevent skin cancer by reducing exposure to ultraviolet radiation: a systematic review. Am J Prev Med 2004; 27(5): 422-66.

22. Hill $D$, Dixon $H$. Promoting sun protection in children: rationale and challenges. Health Educ Behav 1999; 26(3): 409-17.

23. Dobbinson $S$, Wakefield $M$, Hill $D$, et al. Children's sun exposure and sun protection: prevalence in Australia and related parental factors. J Am Acad Dermatol 2012; 66(6): 938-47.

24. Bränström R, Kristjansson $S$, Dal $H$, Rodvall $Y$. Sun exposure and sunburn among Swedish toddlers. Eur J Cancer 2006; 42(10): 1441-7.

25. Dixon $H$, Borland R, Hill D. Sun protection and sunburn in primary school children: the influence of age, gender and coloring. Prev Med 1999; 28(2): $119-30$

26. Tan MG, Nag S, Weinstein M. Parental use of sun protection for their children - does skin color matter? Pediatr Dermatol $2018 ; 35(2): 220-4$

27. Turner LR, Mermelstein RJ. Psychosocial characteristics associated with sun protection practices among parents of young children. J Behav Med 2005; 28(1): 77-90

28. Autier $P$, Doré J-F, Cattaruzza MS, et al. Sunscreen use, wearing clothes, and number of nevi in 6-to 7-year-old European children. J Natl Cancer Inst 1998; 90(24): 1873-80.

29. Geller AC, Colditz G, Oliveria S, et al. Use of sunscreen, sunburning rates, and tanning bed use among more than 10000 US children and adolescents. Pediatrics 2002; 109(6): 1009-14.

30. Reinau D, Achermann C, Arnet N, Meier C, Hatz C, Surber C. Sun protective behaviour of vacationers spending holidays in the tropics and subtropics. Br J Dermatol 2014; 171 (4): 868-74.

31. McLeod GF, Reeder Al, Gray AR, McGee R. Unintended sunburn: a potential target for sun protection messages. J Skin Cancer 2017; 2017: 6902942.

32. Køster B, Thorgaard C, Philip A, Clemmensen IH. Prevalence of sunburn and sun-related behaviour in the Danish population: a crosssectional study. Scan J Public Health 2010; 38(5):548-52.

33. Gefeller $O$, Uter W, Pfahlberg AB. Protection from ultraviolet radiation during childhood: the parental perspective in Bavaria. Int J Environ Res Public Health 2016; 13(10): 1011

34. Li J, Uter W, Pfahlberg A, Gefeller O. A comparison of patterns of sun protection during beach holidays and everyday outdoor activities in a population sample of young German children. Br J Dermatol 2012; 166(4): 803-10.

35. Duarte AF, Picoto $A$, da Costa Pereira $A$, Correia $O$. Sun protection in children: a behavioural study. Eur J Dermatol $2018 ; 28(3): 338-42$
36. Kongsted A, Nielsen AM. Latent class analysis in health research. J Physiother 2017; 63(1): 55-8.

37. Central Committee on Research involving Human Subjects memorandum Behavioural Research, 2002. Available at: file:///D:/ Libraries/Downloads/CCMO+note+Behavioural+research.pdf (accessed 20 Jun 2020).

38. Crutzen R, Ygram Peters G-J, Mondschein C. Why and how we should care about the General Data Protection Regulation. Psychol Health 2019; 34(1 1): 1347-57.

39. TNS-KANTAR. Privacy Policy Online Survey. TNS-KANTAR, 2019. Available at: https://www.tns-nipo.com/getmedia/e03d519b007b-4e77-800a-f737c5eaaa3d/Privacy-Policy-Online-SurveyKantar-TNS-Public-(English).pdf (accessed 20 Jun 2020).

40. Von Elm E, Altman DG, Egger $M$, et al. The strengthening the reporting of observational studies in epidemiology (STROBE) Statement: guidelines for reporting observational studies. Int J Surg 2014; 12(12): 1495-9.

41. Centraal Bureau voor de Statistiek. Standaard Onderwijsindeling. Centraal Bureau voor de Statistiek, 2016. Available at: file:///D:/Libraries/Downloads/PubSoi2016_ed1920.pdf (accessed 11 Aug 2020).

42. Ministry of the Interior and Kingdom Relations, 2020. Available at: https://www.regioatlas.nl/indelingen/indelingen_indeling/ t/provincies (accessed 11 Aug 2020).

43. IBM Corp. IBM SPSS Statistics for Windows, Version 25.0. Armonk, NY: IBM Corp, 2017.

44. Schreiber JB. Latent class analysis: an example for reporting results. Res Social Adm Pharm 2017; 13(6): 1196-201.

45. Cary, NC, USA; SAS Institute Inc., 2020.

46. Gefeller O, Uter W, Pfahlberg AB. Good, but not perfect: parental knowledge about risk factors for skin cancer and the necessity of sun protection in southern Germany. Pediatrc Dermatol 2015; 32(4): e159-60.

47. Thoonen K, van Osch L, Drittij R, de Vries H, Schneider F. A qualitative exploration of parental perceptions regarding children's sun exposure, sun protection and sunburn. Front Public Health 2021; 9:596253.

48. Klostermann S, Bolte G, Group GS. Determinants of inadequate parental sun protection behaviour in their children - results of a crosssectional study in Germany. Int J Hyg Environ Health 2014;217 (2-3): 363-9

49. de Maleissye MF, Beauchet A, Saiag $P$, et al. Sunscreen use and melanocytic nevi in children: a systematic review. Pediatr Dermatol 2013;30(1): 51-9.

50. Gilaberte $Y$, Carrascosa J. Sun protection in children: realities and challenges. Actas Dermosifiliogr 2014; 105(3): 253-62.

51 . Autier $P$, Boniol M, Doré JF. Sunscreen use and increased duration of intentional sun exposure: still a burning issue. Int J Cancer 2007; 121 (1): 1-5.

52. O'Riordan DL, Nehl E, Gies $P$, et al. Validity of covering-up sunprotection habits: association of observations and self-report. J Am Acad Dermatol 2009; 60(5): 739-44.

53. Schlarbaum JP, Lazovich D, Dodd E, Hanson B, Polcari IC. Examination of use and barriers for five sun protection strategies in parents and their children. Pediatr Dermatol 2020;37(5): 827-32.

54. Schneider S, Bolbos A, Kadel P, Holzwarth B. Exposed children, protected parents; shade in playgrounds as a previously unstudied intervention field of cancer prevention. Int J Environ Health Res 2020; 30(1): 26-37.

55. Adams MA, Mayer JA, Bowen DJ, Ji M. Season of interview and self-report of summer sun protection behaviors. Cancer Causes Control 2009; 20(2): 153-62

56. Dobbinson SJ, Jamsen K, Dixon HG, et al. Assessing populationwide behaviour change: concordance of 10 -year trends in self-reported and observed sun protection. Int J Public Health 2014;59(1): $157-66$ 
57. Køster B, Søndergaard J, Nielsen J, Allen M, Olsen A, Bentzen $J$. The validated sun exposure questionnaire: association of objective and subjective measures of sun exposure in a Danish population-based sample. Br J Dermatol 2017; 176(2): 446-56.

58. Hubbard G, Cherrie J, Gray J, et al. Sun protection education for adolescents: a feasibility study of a wait-list controlled trial of an intervention involving a presentation, action planning, and SMS messages and using objective measurement of sun exposure. $B M C$ public health 2020; 20(1): 131.

59. Glanz K, Gies P, O'Riordan DL, et al. Validity of self-reported solar UVR exposure compared with objectively measured UVR exposure. Cancer Epidemiol Biomarkers Prev 2010; 19(12): 3005-12.

60. Vanos JK. Children's health and vulnerability in outdoor microclimates: a comprehensive review. Environ Int 2015; 76: 1-15.
61. Termorshuizen F, Wijga A, Garssen J, Den Outer PN, Slaper H, Van Loveren $\mathrm{H}$. Exposure to solar ultraviolet radiation in young Dutch children: assessment by means of a 6-week retrospective questionnaire. J Expo Sci Environ Epidemiol 2002; 12(3): 204-13.

62. Thoonen K, Osch Lv K, Vries Hd K, Jongen S, Schneider F. Are environmental interventions targeting skin cancer prevention among children and adolescents effective? A systematic review. Int J Environ Health Res 2020; 17(2): 529.

63. Christian H, Lester L, Trost SG, et al. Shade coverage, ultraviolet radiation and children's physical activity in early childhood education and care. Int J Public Health 2019; 64(9): 1325-33.

64. Bellamy R. A systematic review of educational interventions for promoting sun protection knowledge, attitudes and behaviour following the QUESTS approach. Med Teacher 2009; 27(3): 269-75. 